Revue des patrimoines

$36 \mid 2018$

Les archives photographiques de presse, pratiques comparées et enjeux méthodologiques

\title{
L'exemple du musée Nicéphore Niépce (Chalon- sur-Saône) : conservation, documentation et valorisation des photographies de presse
}

Conservation and exploitation of newspaper photos, the example of the Nicéphore Niépce museum at Chalon-sur-Saône

\section{Sylvain Besson}

\section{(2) OpenEdition}

\section{Journals}

Édition électronique

URL : http://journals.openedition.org/insitu/18361

DOI : 10.4000/insitu.18361

ISSN : 1630-7305

Éditeur

Ministère de la culture

\section{Référence électronique}

Sylvain Besson, «L'exemple du musée Nicéphore Niépce (Chalon-sur-Saône) : conservation, documentation et valorisation des photographies de presse », In Situ [En ligne], 36 | 2018, mis en ligne le 15 octobre 2018, consulté le 21 avril 2019. URL : http://journals.openedition.org/insitu/18361 ; DOI : 10.4000/insitu. 18361

Ce document a été généré automatiquement le 21 avril 2019.

\section{c)}

In Situ Revues des patrimoines est mis à disposition selon les termes de la licence Creative Commons Attribution - Pas d'Utilisation Commerciale - Pas de Modification 4.0 International. 


\title{
L'exemple du musée Nicéphore Niépce (Chalon-sur-Saône) : conservation, documentation et valorisation des photographies de presse
}

\author{
Conservation and exploitation of newspaper photos, the example of the \\ Nicéphore Niépce museum at Chalon-sur-Saône
}

Sylvain Besson

1 Le musée Nicéphore Niépce s'est imposé depuis sa création en 1974 comme le lieu de conservation, d'étude et de valorisation des fonds photographiques les plus divers, de l'invention du procédé par Nicéphore Niépce aux dernières avancées du numérique. La collection est riche de plus de trois millions de phototypes, 6000 appareils photographiques, 30000 livres de photographies et 40000 revues illustrées par la photographie ou sur la photographie. Le musée Nicéphore Niépce s'attelle à rendre compte de la diversité du photographique et de sa pratique, au sein de son parcours permanent, dans des expositions temporaires et sur Internet. Que ce soit par la présentation d'originaux ou via des installations multimédias, le musée Nicéphore Niépce renouvelle l'étude et la valorisation de la photographie.

2 La presse illustrée par la photographie occupe une place centrale dans les collections du musée qui a acquis, depuis une vingtaine d'années, de nombreux magazines illustrés, les intégrant à ses collections et les traitant comme telles. En parallèle, le musée s'est attaché à acquérir des fonds de photographes ou d'agences ayant travaillé pour la presse. L'étude de ces différentes « catégories » de collections assure une meilleure compréhension de la pratique des photographes et de leurs commanditaires. 


\section{Des collections de magazines, des archives d'auteurs} d'acquisition volontaire, le musée Nicéphore Niépce a enrichi progressivement la collection avec des magazines essentiels pour la compréhension de l'histoire des revues illustrées par la photographie et des modes de production des images. Dans la plupart des cas, les collections sont complètes (C) :

4 - Le Miroir (C),

\section{Étude - indexation}

Traditionnellement, le musée Nicéphore Niépce utilise les normes d'indexation préconisées par Joconde ${ }^{1}$ pour décrire ses collections. L'iconographie est analysée avec le thesaurus Garnier ${ }^{2}$. Cependant, les volumes à indexer étant particulièrement importants, le choix a été fait de se concentrer sur les champs les plus interrogés, à savoir titre, auteur, date, technique, iconographie.

Pour les magazines illustrés, une description plus détaillée a été élaborée. Le protocole diffère d'un magazine à l'autre; il s'adapte à la fois aux nécessités d'indexation incompressibles détaillées plus haut, à la nature même de l'objet magazine, qui combine texte et image, et à l'exploitation future pressentie de la description. 

les tris et les recherches croisées par photographe. Il donne également la possibilité de
rendre prégnante la répartition photos d'agence/photos d'auteur ou d'analyser les
associations photographe/rédacteur de l'article, par exemple.

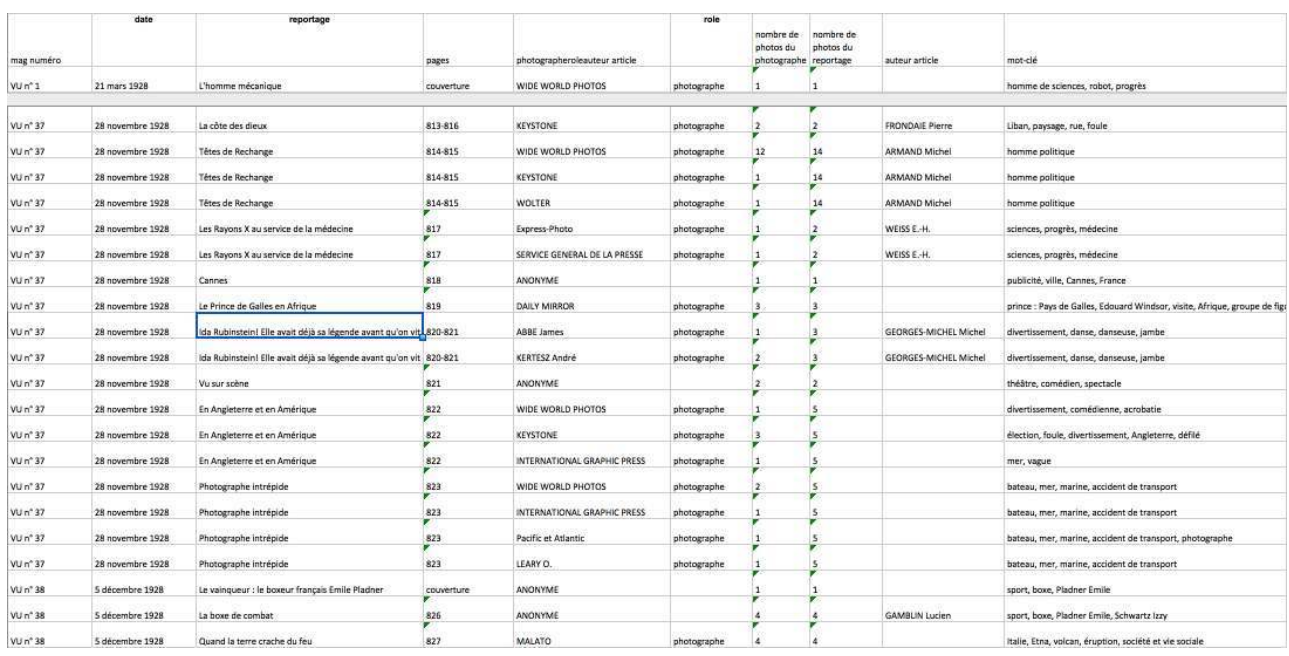

Dans le cadre du projet «PhotoCréation» financé par l'Agence nationale pour la recherche (ANR) piloté par l'historien de la photographie Michel Frizot, le musée a expérimenté différents types d'indexation selon les magazines, entre 2008 et 2012.

Pour les magazines VU (1928-1940, fondé par Lucien Vogel) et Art et Médecine (1929-1939, créé par François Debat), sous Excel, l'indexation a été réalisée de manière à créer un outil de recherche exploitable par tous mais le plus fin possible. L'objectif était de rendre sensible une des caractéristiques principales de ces deux magazines: la présence d'auteurs photographes, crédités en tant que tels, au détriment des agences et des grands studios. Chaque reportage a bénéficié d'un traitement à part, se voyant accorder une ligne descriptive pour chaque photographe ayant une photographie reproduite dans l'article.

Les champs renseignés sont les suivants :

- numéro du magazine,

- date de publication,

- nom du reportage,

- nom de l'auteur du rédactionnel,

- nom de l'auteur des photographies,

- nombre de photos du reportage,

- nombre de photos par le photographe,

- description iconographique globale des images du reportage.

Concrètement, un reportage composé de clichés produits par trois photographes sera traité dans trois lignes Excel différentes. Chacune des lignes sera identique, elles seront distinguées par le nom des photographes et le nombre de leurs photographies publiées dans le reportage.

Une fois l'indexation de l'intégralité du magazine achevée, ce type de traitement permet Une fois lindexation de lintegralté du magazine achevée, ce type de traitement permet associations photographe/rédacteur de l'article, par exenple.

Par ailleurs, l'intégralité des magazines ont été scannés et ont été soumis à un traitement $\mathrm{OCR}^{3}$ de manière à pouvoir effectuer des recherches de mots directement dans les pages numérisées. 

de la photographie, de l'invention du procédé par Nicéphore Niépce (1765-1833) aux dernières avancées du numérique selon différents points de vue sur le médium: technique, commercial, publicitaire... L'utilisation de la photographie par la presse et l'édition au cours $\mathrm{du} \mathrm{xx}^{\mathrm{e}}$ siècle fait l'objet de présentations régulières dans l'exposition permanente. Les approches sont multiples et diffèrent d'un accrochage à l'autre au gré des rotations. Il s'agit, sur une seule cimaise, d'aborder un aspect des relations entre la presse illustrée et les photographies.

34 À titre d'exemple, afin d'évoquer les difficultés pour un journal quotidien $\mathrm{du} \mathrm{xx}^{\mathrm{e}}$ siècle d'intégrer des photographies, les tirages retouchés avant impression (pour augmenter les contrastes) issus des archives du Petit Parisien sont exposés à côté de la double-page correspondante.

35 A partir d'un exemple de collaboration entre le photographe Jean Moral (1906-1999) et un magazine, en l'occurrence le magazine de mode Harper's Bazaar, le musée a souhaité mettre l'accent sur la pluralité des objets photographiques nécessaires à l'élaboration du travail éditorial : les planches-contacts (de manière à montrer le reportage complet), les tirages de lecture (avec ou sans indications de recadrage ou de retouche) et les doublespages du magazine afférent. Un appareil photographique semblable à celui utilisé par Moral permet au visiteur de prendre la mesure de la démarche du photographe.

Dans le parcours permanent, des cimaises non spécifiquement dédiées aux relations entre photographie et presse illustrée incluent/comprennent souvent des magazines. Ainsi en est-il de la cimaise "La photographie, un objet esthétique et social», qui accueille régulièrement des images de presse ou des magazines. La photographie a été un des moyens privilégiés pour transmettre les bienfaits des congés payés en 1936 dans VU, Regards ou Marianne, grâce aux photographies de Jean Moral, André Steiner (1901-1978) et d'autres (fig. 1, fig. 2). 
Figure 1

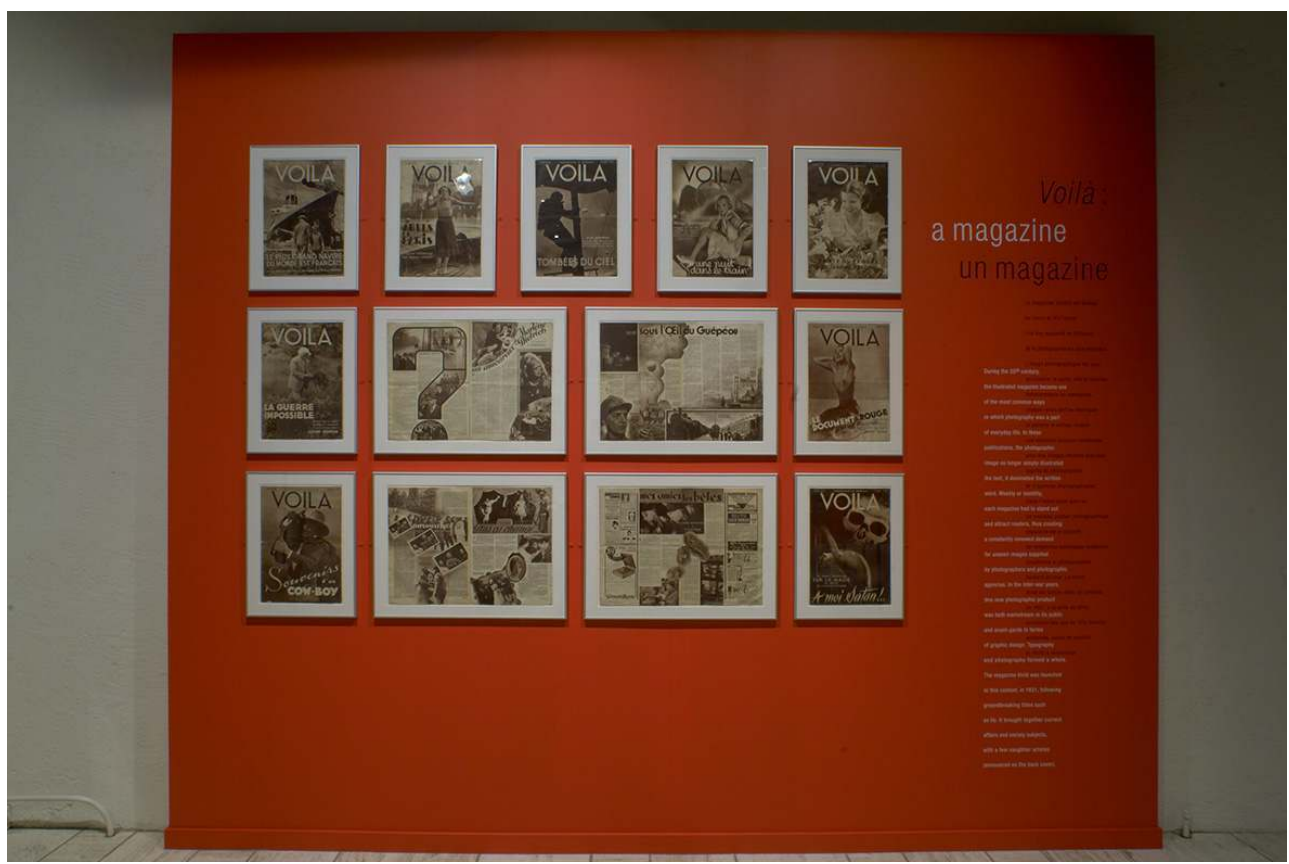

Vue de la salle Durville, cimaise consacrée au magazine Voilà.

(c) Musée Nicéphore Niépce, ville de Chalon-sur-Saône.

Figure 2

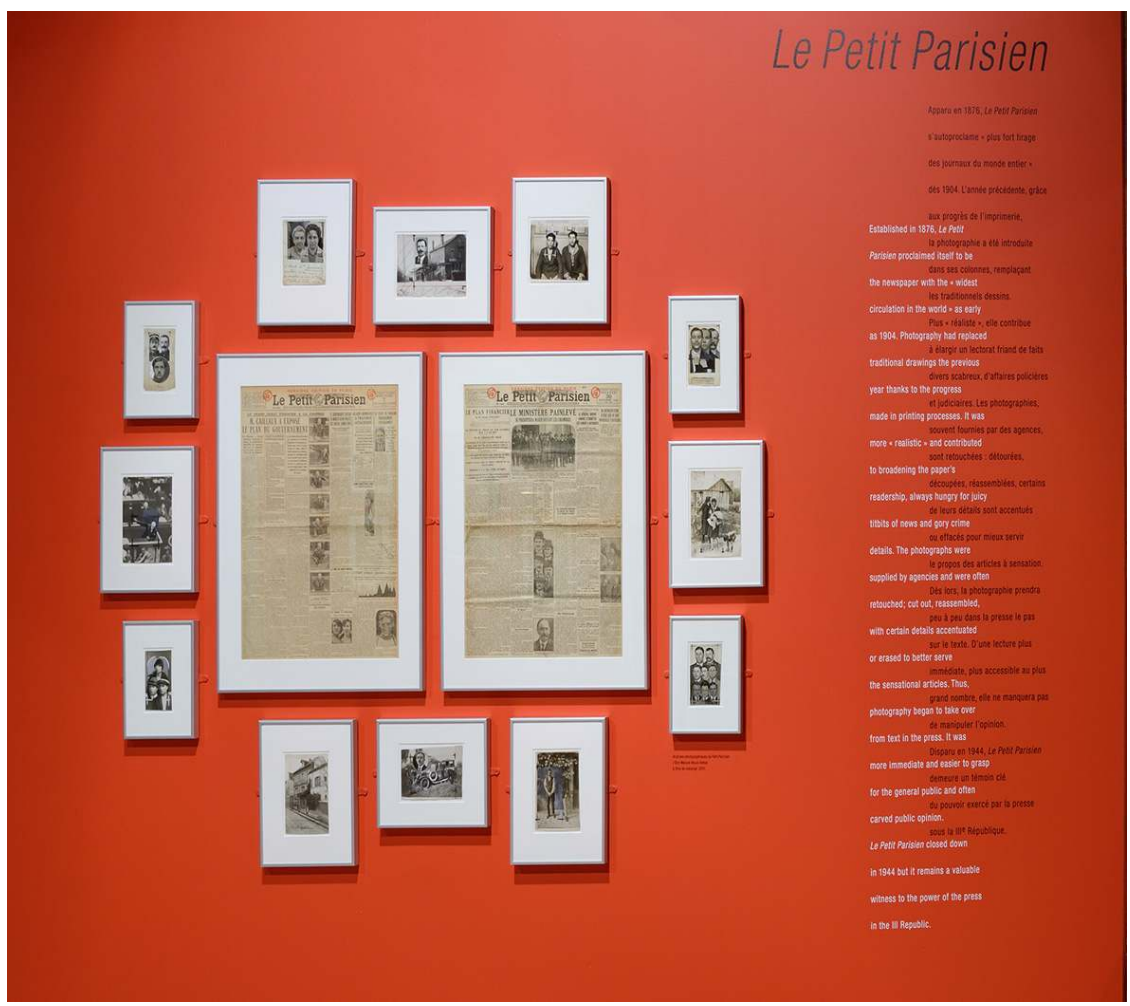

Vue de la salle Durville, cimaise consacrée au journal Le Petit Parisien.

(c) Musée Nicéphore Niépce, ville de Chalon-sur-Saône. 


\section{Valorisation par l'exposition temporaire}

Les expositions temporaires sont des moments privilégiés pour mettre en avant la photographie de presse, que ce soit dans des expositions entièrement consacrées à un magazine ou dans des expositions où le magazine illustré est un des moyens utilisés pour expliciter tel ou tel aspect du propos.

A ce titre, l'exposition " $V U$, un magazine photographique $"^{5}$ fait figure de référence. Coproduite par le musée Nicéphore Niépce et la Maison européenne de la photographie (MEP), elle a été présentée en premier lieu à Paris (2006) puis à Chalon-sur-Saône (2007). Les commissaires Michel Frizot et Cédric de Veigy ont conçu et réalisé l'exposition à partir des seules collections du musée.

VU est le premier magazine d'actualité entièrement photographique. Publié entre 1928 et 1940, VU invente les notions de photoreportage et renforce le statut des auteurs photographes. Il se singularise par l'utilisation de la rotogravure ${ }^{6}$ qui accorde une plus grande souplesse dans la mise en page et souligne les points de vue nouveaux recherchés par les photographes. L'exposition retraçait l'histoire du magazine, en présentait ses différents acteurs, les innovations techniques et intellectuelles induites par ce magazine, par l'exposition exclusive de couvertures et de doubles-pages. Les archives du magazine ne pouvaient être montrées: elles ont disparu durant la Seconde Guerre mondiale. Cependant, l'impact des couvertures, conçues par son directeur artistique Alexander Liberman (1912-1999), le dynamisme des doubles-pages, la force du parti-pris éditorial tellement évident devant les originaux, compensaient largement cette absence.

Les magazines étaient présentés soit en vitrine, soit dans des boîtes-papillon ${ }^{7}$ permettant d'isoler telle ou telle double-page, soit au mur, protégées par des plexis lorsque plusieurs couvertures ou doubles-pages étaient associées (fig. 3, fig. 4). 
Figure 3

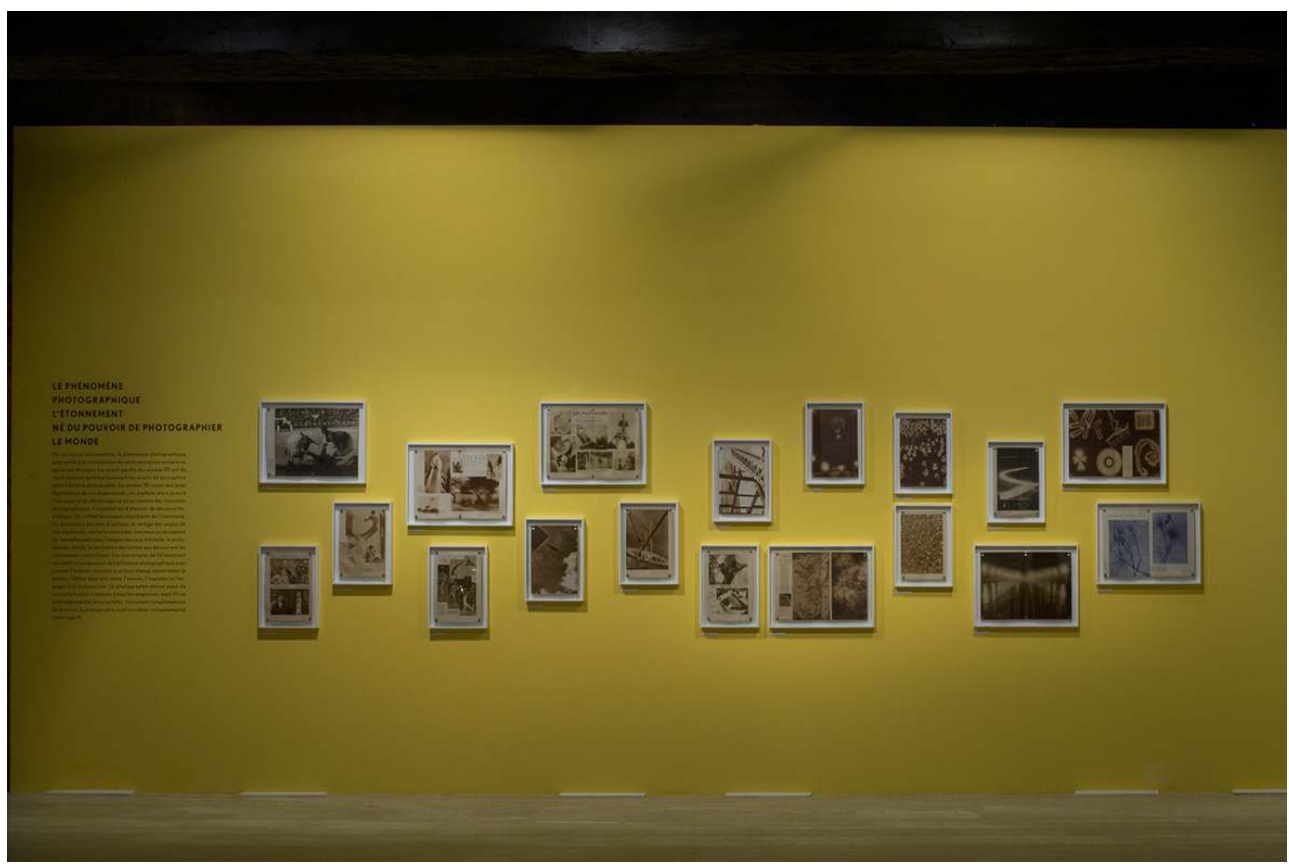

Vue de l'exposition « Regarder VU », 2007.

(c) Musée Nicéphore Niépce, ville de Chalon-sur-Saône.

Figure 4

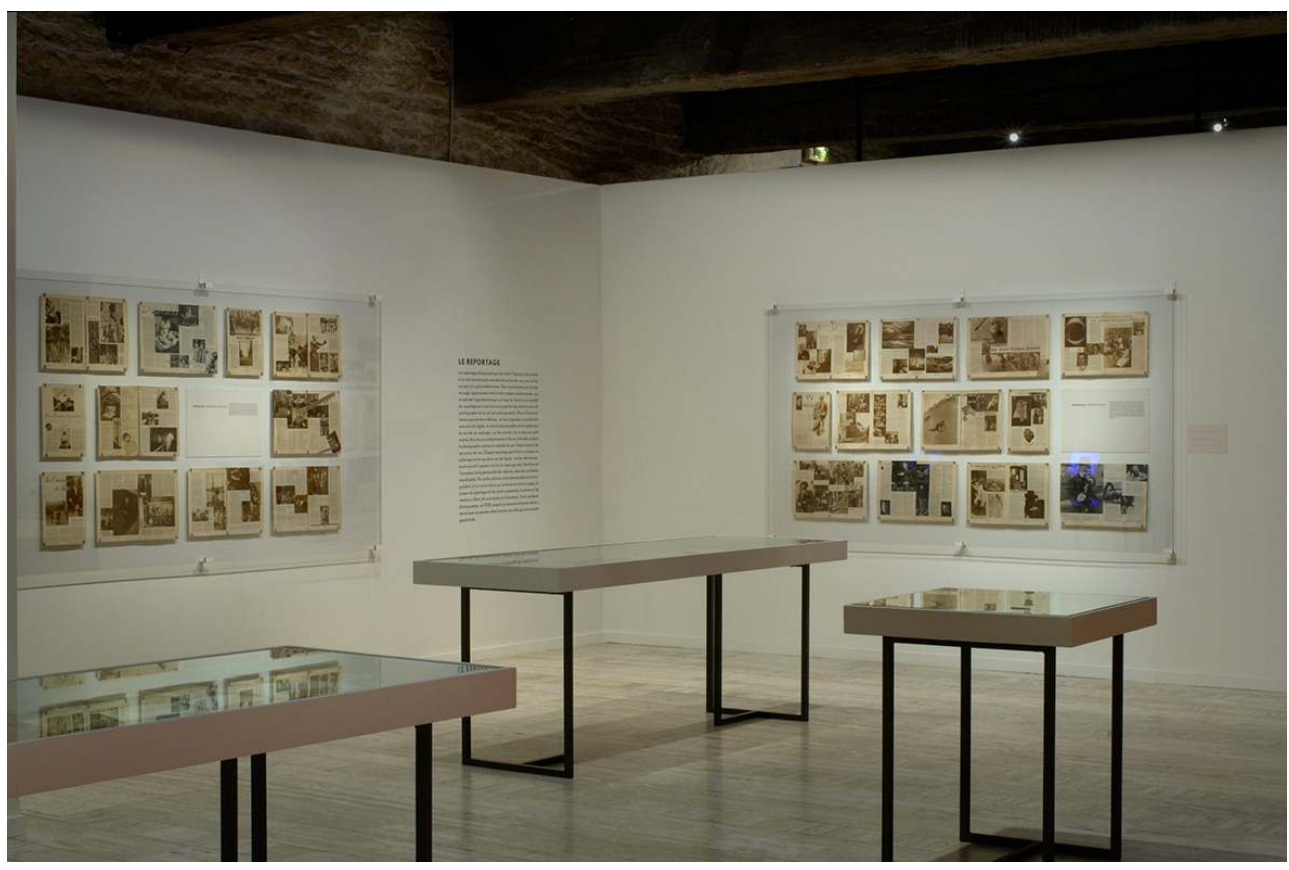

Vue de l'exposition « Regarder VU », 2007.

(c) Musée Nicéphore Niépce, ville de Chalon-sur-Saône.

41 Plus souvent, les expositions historiques du musée Nicéphore Niépce présentent à la fois tirages et magazines, ces derniers soutenant le propos et permettant de comprendre le 
contexte de création des photographies, ainsi que la manière de les diffuser au public au moment de leur publication.

L'exposition "L'Ivresse du mouvement", produite en 2016, était consacrée à la photographie de sport. Elle présentait des photographies de Jean Moral, André Steiner, Pierre Boucher (1908-2000) ou Isaac Kitrosser (1899-1984) à côté des magazines Marianne, VU, Voilà, Match L'Intran, La Vie au grand air.

La double-page du reportage d'Isaac Kitrosser pour Marianne était encadrée, placée au centre de la cimaise. Autour d'elle étaient disposés, encadrés, les différents tirages du reportage. Les exemplaires de La Vie au grand air du musée sont reliés, ils étaient présentés en vitrine. Les autres magazines étaient encadrés et cohabitaient au mur avec des tirages.

L'exposition «L'Indochine en guerre, des images sous contrôle ", produite en 2006, faisait de constants allers-retours entre photographies et magazines et illustrait la dichotomie entre les reportages produits par les photoreporters et les images publiées dans la presse (Regards, Paris-Match), une fois passées au filtre de la censure (fig. 5).

Figure 5

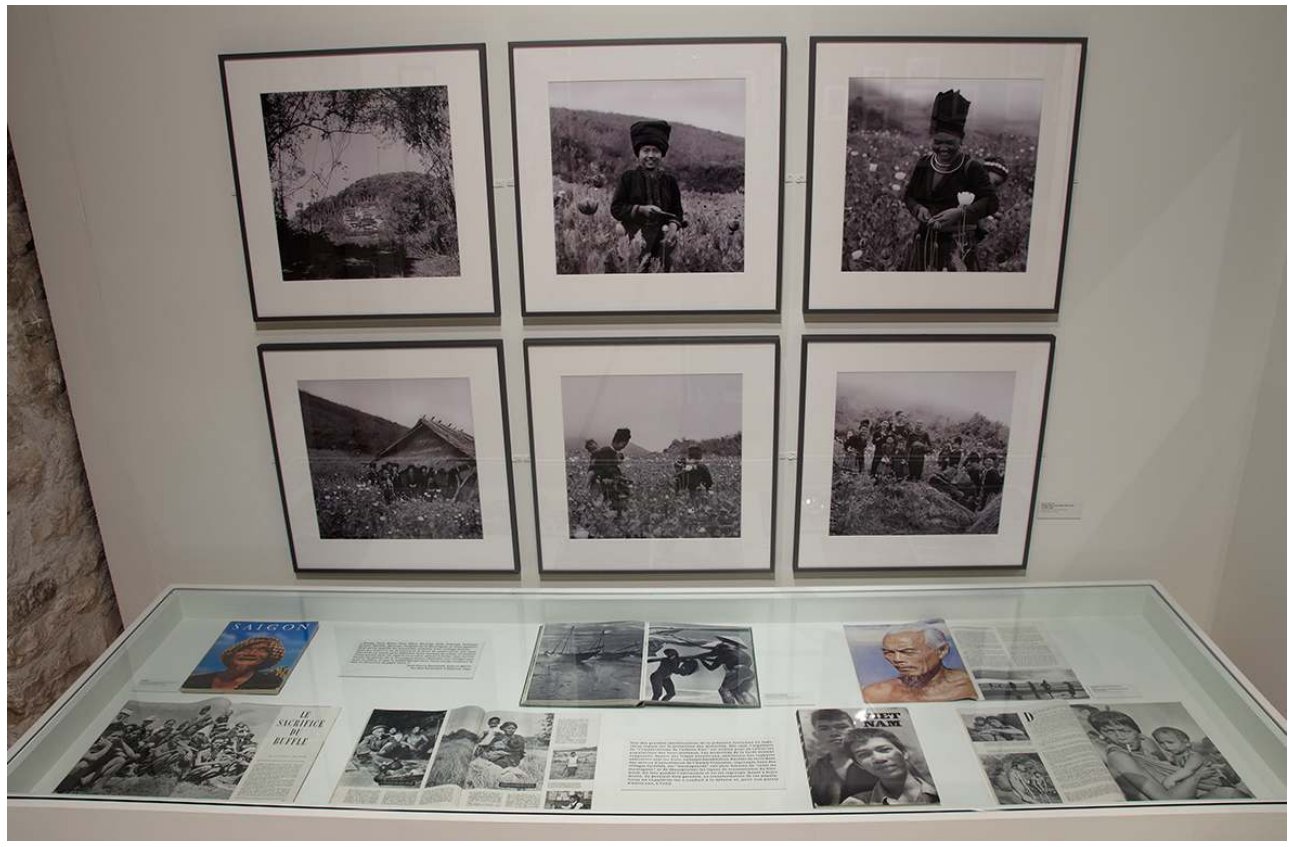

Vue de l'exposition «L'Indochine en guerre, l'image sous contrôle, 1945-1954 », 2010.

(c) Musée Nicéphore Niépce, ville de Chalon-sur-Saône.

Présentée en 2009, la rétrospective consacrée à Peter Knapp (1931- ) n'aurait pu se passer des numéros de Elle dont il fut le directeur artistique de nombreuses années, exposés aux côtés de ses planches-contacts pour le couturier Courrèges et ses travaux de plasticien.

"Le Chic français : images de femmes, 1900-1950 ", produite par la Ville d'Évian à partir des collections du musée Nicéphore Niépce et présentée au Palais Lumière d'octobre 2017 à janvier 2018, souligne combien l'utilisation massive de la photographie dans les magazines de mode à partir des années 1920 a bénéficié de la concomitance de l'apparition du concept de haute couture, de l'émergence de photographes indépendants de talent et des efforts déployés par certains éditeurs de presse pour démocratiser la 
mode tout en installant Paris comme sa capitale. Sur les cimaises, les travaux avantgardistes de Maurice Tabard (1897-1984), Man Ray (1890-1976), Germaine Krull (1897-1985), André Steiner, Jean Moral (des tirages mais également des magazines généralistes tels Paris-Magazine, La France travaille, VU, Diversion) cohabitent avec les épreuves qu'ils publient dans les magazines de mode phares de l'époque (Le Jardin des modes, Harper's Bazaar, Marie Claire, etc.). Les magazines sont alternativement exposés dans des vitrines ou encadrés, sur les murs. Ainsi, l'exposition propose une section Jardin des modes et une autre Marie Claire: ces deux magazines sont encadrés, que l'on montre des couvertures ou des doubles-pages. Les Harper's Bazaar, plus fragiles, sont dans des vitrines.

Un autre aspect de l'exposition souligne le réemploi par un photographe des images qu'il va publier dans plusieurs titres de presse, dans des contextes différents. La planchecontact du reportage commandé par Harper's Bazaar à Jean Moral en 1933 au cap de Formentor (Majorque), dans la demeure du dessinateur de mode Reynaldo Luza (1893-1978), est présentée encadrée. En regard, une vitrine expose les multiples publications comportant des images extraites de ce reportage (plusieurs numéros de Paris-Magazine, Diversion, $\mathrm{Vu}$ ).

Les structures métalliques noires, supports d'agrandissements de couvertures de magazines et qui organisent les espaces, le design des vitrines, les bandes noires habillant les textes de salle rappellent la composition des doubles-pages et la table de travaille du graphiste qui compose sa double-page. L'exposition tente de traduire par sa scénographie, son mobilier et le format des cadres les allers-retours permanents entre direction artistique des magazines, choix des photographies, agencement entre elles dans la double-page et parti-pris rédactionnel.

\section{Valorisation par la mise en ligne}

Pour le magazine Le Miroir, dont il conserve une collection complète, le musée Nicéphore Niépce souhaitait mettre en ligne l'intégralité de sa collection pour la période de la Première Guerre mondiale. Il a pour ce faire bénéficié du soutien du ministère de la Culture dans le cadre d'un appel à projet sur les «Services numériques culturels innovants » en 2012 et s'est associé à un porteur de projet, Nicéphore Cité et On-Situ, une entreprise spécialisée dans les dispositifs de médiation.

Le Miroir est un magazine qui a posé les jalons de la future presse illustrée d'actualité. Durant la période de la guerre 1914-1918, le magazine devient entièrement photographique (15 pages sur 16). Cependant, le traitement des faits est entièrement factuel, sans filtre, ce que la mise en page retranscrit. Chaque page est structurée avec un titre général, une légende-titre par demi-page, une légende descriptive assez longue pour chaque photographie ou groupe de photographies. Les auteurs ne sont pas crédités (textes et images). Plusieurs effets photographiques sont mis en avant, tel l'instantané, les photographies sont largement retouchées. Il s'agit, au fil des publications hebdomadaires, de suivre la guerre au jour le jour, avec une semaine de décalage, sans qu'un événement particulier ne prenne le pas sur un autre, et de rendre compréhensible l'envergure mondiale du conflit. Chaque semaine, les lecteurs (et ils sont nombreux, un million de lecteurs hebdomadaires revendiqué !) découvrent le monde en images, certes sous le prisme de la guerre; ils "voient" pour la première fois des lieux inaccessibles et inconnus, et dans des circonstances exceptionnelles. 
51 Pour le musée Nicéphore Niépce, le dispositif en ligne devait rendre compte de tous ces éléments: la mise en page, le caractère mondial du conflit, l'usage intensif de la photographie. Avec On-Situ, la réflexion fut donc menée sur deux axes, tant sur la forme que le fond.

52 L'indexation a tenu compte de ces impératifs. Après avoir numérisé et océrisé les quelque 250 numéros concernés, le musée Nicéphore Niépce a procédé à l'indexation des photographies une par une sous Excel avec, pour chaque bloc de photographies :

53 - numéro du magazine,

54 - date,

55 - titre de la page,

56 - légende-titre,

57 - légende descriptive,

58 - mots clefs iconographiques,

59 - localisation la plus précise possible : pays/région/commune/lieu-dit,

60 - personnages identifiés.

61 Le site mis à disposition du public tente, dès l'ouverture, de rendre compte du caractère mondial du conflit: une mappemonde occupe l'intégralité de l'écran. Une frise chronologique figure au bas de l'écran, qui permet de choisir le magazine par date. L'utilisateur n'a pas accès immédiatement au magazine choisi : sur la mappemonde apparaissent les titres des articles du magazine sélectionné localisés sur les lieux concernés. En cliquant sur un titre, la frise chronologique est remplacée par une nouvelle frise contenant le chemin de fer du magazine. La mappemonde disparaît au bénéfice de la double-page correspondant au choix de l'internaute. Ce dernier peut dès lors faire défiler chaque double-page du numéro sélectionné pour la voir agrandie.

Une seconde partie du site donne accès à une partie rédactionnelle qui présente en huit sections ce qui différencie Le Miroir de ses concurrents et caractérise la singularité de son programme éditorial (fig. 6, 7, 8). 
Figure 6

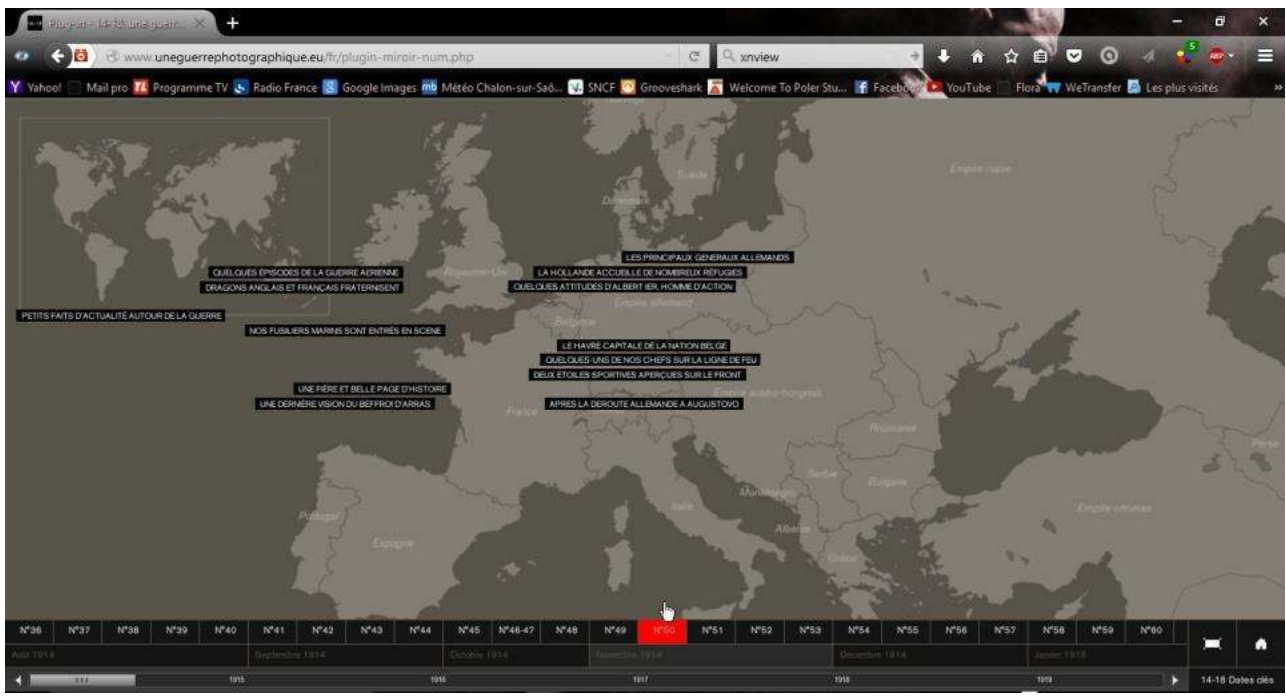

Copie d'écran du site : www.uneguerrephotographique.eu.

(c) Musée Nicéphore Niépce, ville de Chalon-sur-Saône.

Figure 7

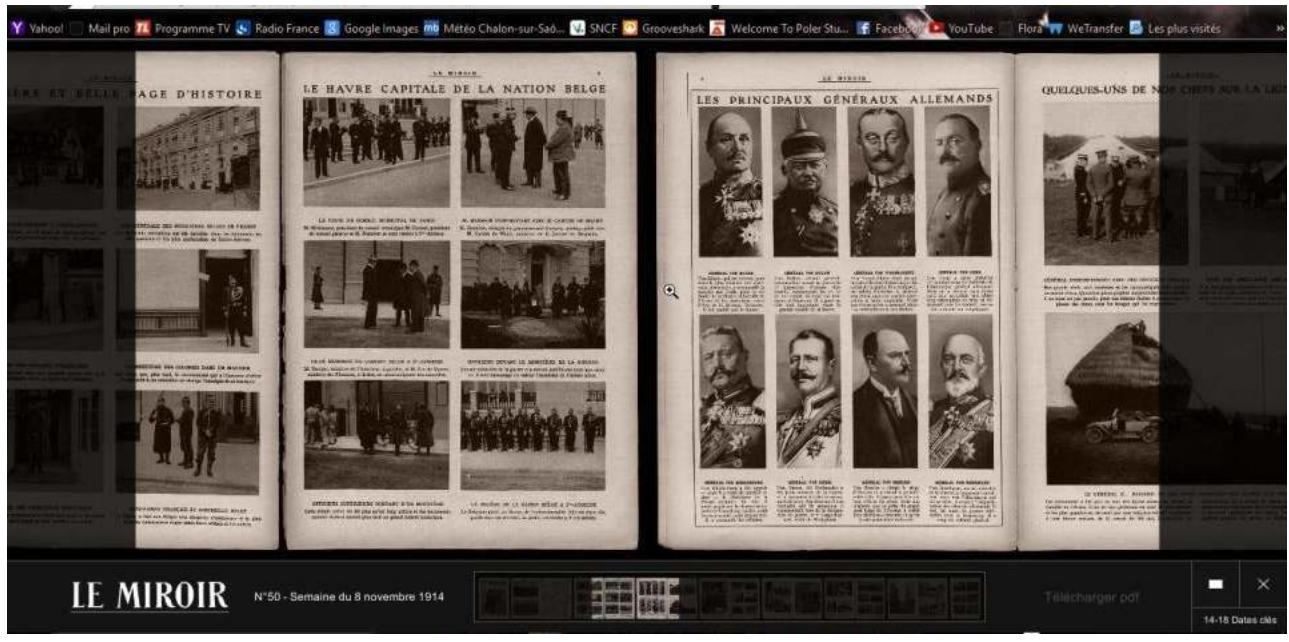

Copie d'écran du site : www.uneguerrephotographique.eu.

(c) Musée Nicéphore Niépce, ville de Chalon-sur-Saône. 


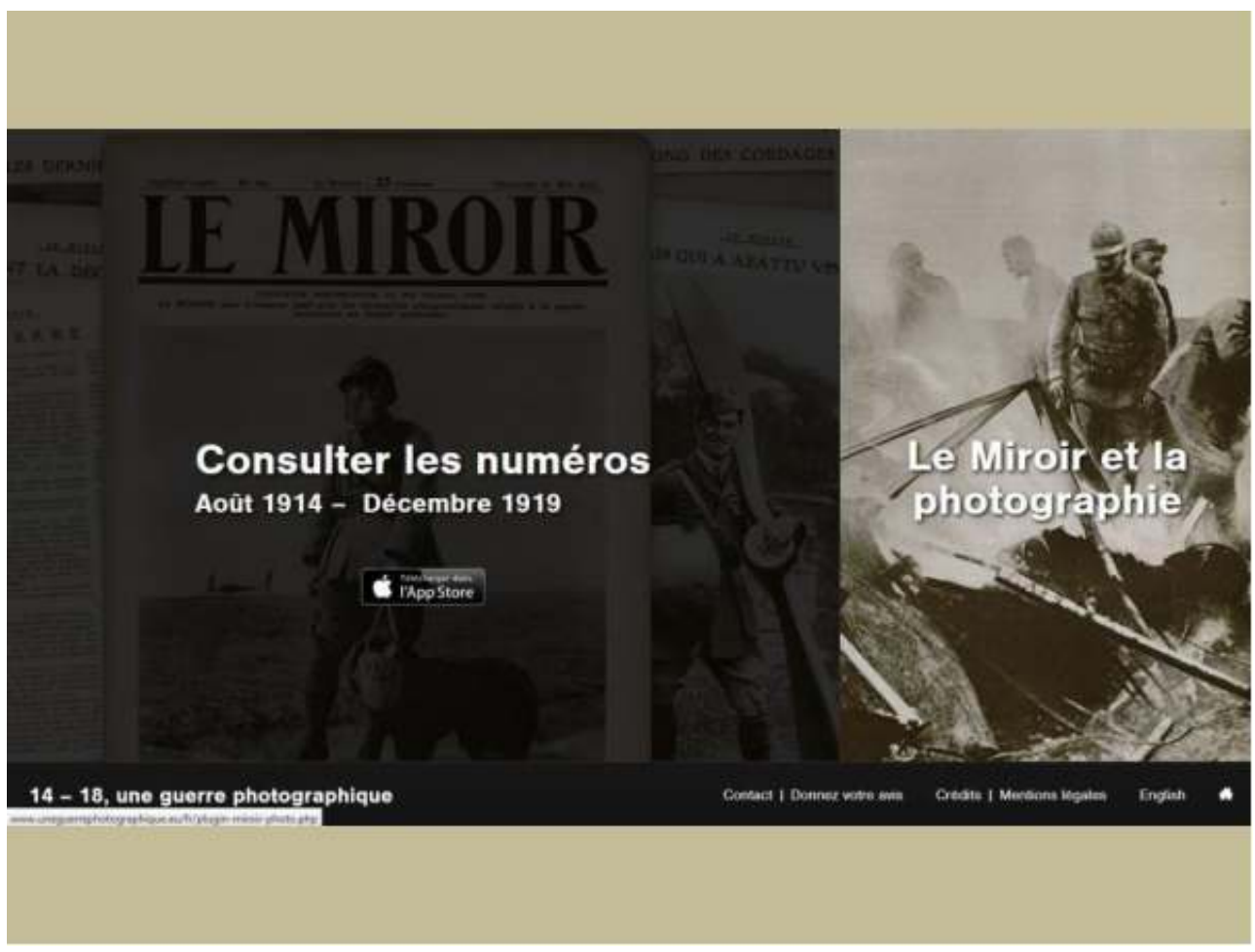

Copie d'écran du site : www.uneguerrephotographique.eu.

(c) Musée Nicéphore Niépce, ville de Chalon-sur-Saône.

\section{Valorisation des fonds photographiques de presse par des dispositifs de médiation innovants}

Dans le cadre du programme CONTINT (Contenus et Interactions ${ }^{8}$ ) 2012 de l'ANR, le musée Nicéphore Niépce s'est associé à un porteur de projet, Nicéphore Cité, deux laboratoires de recherche ( $\mathrm{du}^{\mathrm{CNAM}}{ }^{9}$ et de l'IGN $^{10}$ ) spécialisés dans les algorithmes de reconnaissance automatique d'image et On-Situ, une entreprise spécialisée dans les dispositifs de médiation afin de créer l'application POEME (Environnement immersif pour l'exploration de contenus photographiques).

64 Les fonds photographiques du musée Nicéphore Niépce, sa base de données et les fichiers numériques ont constitué le matériau premier pour l'expérimentation et l'amélioration des algorithmes développés par le CNAM et l'IGN. On-Situ était responsable de l'élaboration d'un dispositif de consultation des fonds du musée intégrant les recherches textuelles habituelles du musée et les algorithmes d'automatisation du CNAM et de l'IGN.

Une des principales motivations était l'aide à l'identification des multiples d'une photographie au sein d'un large corpus d'images plus ou moins homogène, en couplant la recherche textuelle et les algorithmes. Par exemple, dans le fonds Jean Moral, nous souhaitions, partant d'un tirage, repérer plus facilement les planches-contacts correspondantes, le négatif, les différentes variantes (contact $6 \times 6$, tirages de lecture dans différents formats) ainsi que les publications afférentes. Ce travail ayant déjà été 
réalisé «manuellement », les algorithmes ont pu être testés sur un matériau connu, bien identifié et référencé durant les trois ans de recherche et développement de POEME.

Si les algorithmes sont encore perfectibles, si l'indexation manuelle est fortement dépendante du temps que l'on peut y consacrer et, dans le cas d'analyse pointue de fonds de photographes, forcément tributaire de la capacité des chercheurs et documentalistes à mémoriser un grand nombre d'images pour les lier entre elles, la réflexion sur la combinaison des deux a abouti à la mise en place d'un dispositif de consultation et de recherche résolument différent et innovant.

On-Situ et le musée Nicéphore Niépce avaient déjà collaboré pour créer des dispositifs de consultation de fonds photographiques volumineux. "Corpus» donnait accès à l'intégralité de la collection de Bernard Lamarche-Vadel (1949-2000) (1 800 numéros) dans un espace immersif. "Surface en Relief », proposé dans le parcours permanent du musée Nicéphore Niépce, permet la consultation de quelque 3000 photographies stéréoscopiques des collections, de manière collective, chaque série étant commentée. « À l'œil », conçu autour des collections de photographies anonymes du musée, rendait compte de la diversité des fonds photographiques du musée dans le cadre d'une exposition temporaire. POEME a donné l'occasion de réfléchir autrement à ce type d'interface. Certains principes des dispositifs précédents sont toujours présents: consultation collective sur un écran "panoramique» et immersif, liberté pour le spectateur de construire sa propre recherche sans dépendre du langage, technique, de description des images par le musée. Avec POEME, le musée Nicéphore Niépce va plus loin dans l'interactivité entre les spectateurs et la base de données, et dans le détachement vis-à-vis des systèmes descriptifs professionnels : projection géante de plus de $180^{\circ}$ pour « voir» les résultats d'une recherche, table tactile interactive où le spectateur construit sa recherche en choisissant les mots-clefs, en utilisant les algorithmes mathématiques ou en couplant les deux modes de recherche.

68 L'aboutissement du projet POEME fut sa présentation à Paris Photo en 2016 avec un test grandeur nature par l'ensemble des professionnels du milieu de la photographie pendant cinq jours. Toujours en développement, POEME propose néanmoins un système inédit de consultation d'archives photographiques, ouvrant la voie à un retournement de son objectif initial: proposer aux iconographes de nouveaux modes de consultation afin d'éditer des images en vue de leur publication.

\section{Conclusion}

69 Le musée Nicéphore Niépce mène depuis plusieurs années une réflexion autour de la presse illustrée par la photographie. Cela se traduit par la constitution de fonds de référence autour d'un certain nombre de titres qui ont fait date dans l'histoire de la presse illustrée couplée à un travail d'indexation et de numérisation approfondis, accessible à tous en ligne. Cela se traduit également par l'accueil de fonds complets de photographes ayant travaillé pour la presse. Le musée conserve ainsi toutes les étapes de vie d'une image : du négatif à la planche-contact, du tirage de lecture à l'editing définitif jusqu'à la publication.

70 Ces collections bénéficient de présentations ponctuelles au sein du parcours permanent ou dans des expositions temporaires qui mettent en avant le rôle des magazines dans la vie et la démarche des photographes. Surtout, cette « matière première » volumineuse et 
variée donne à réfléchir pour inventer de nouveaux modes de restitution au public et aux professionnels. Le musée Nicéphore Niépce devient lieu d'expérimentation, les archives de presse y gagnent en visibilité et sont traitées à l'égal des œuvres de plasticiens habituellement mises en avant dans les musées.

\section{NOTES}

1. - Catalogue collectif des collections des musées de France, ministère de la Culture : http:// www.culture.gouv.fr/documentation/joconde/fr/partenaires/AIDEMUSEES/inventaireinformatise.htm.

2. - À partir du Thesaurus iconographique. Système descriptif des représentations de François Garnier. Paris : Le Léopard d'or, 1984. Voir le site : http://www2.culture.gouv.fr/documentation/joconde/ $\mathrm{fr} /$ partenaires/AIDEMUSEES/thesaurus-garnier/thesaurus-pres.htm.

3. - Optical caracter recognition ou reconnaissance optique de caractères.

4. - Voir les sites : www.museeniepce.com/index.php?/collections/enjeux-de-la-photographie/ $\mathrm{vu}$ et www.museeniepce.com/index.php?/collections/enjeux-de-la-photographie/art-etmedecine. Le musée Nicéphore Niépce a également mis en ligne les 230 premiers numéros de Voilà, sans leur indexation: www.museeniepce.com/index.php?/collections/enjeux-de-laphotographie/Voila [consultés le 24/08/2018].

5. - Voir le site: http://www.museeniepce.com/index.php?/collections/enjeux-de-laphotographie/vu [consulté le 24/08/2018].

6. - Rotogravure: procédé d'impression de gravure en creux, où la plaque d'impression est montée sur cylindre. Les deux insolations successives (la maquette réalisée sur une table lumineuse - donnant une plus grande liberté de mise en page - insolée sur un papier charbon photosensible, puis le papier charbon sur la trame de l'héliogravure) garantissent le meilleur rendu des dégradés des photographies originales.

7. - Boîte-papillon : le magazine est posé sur un tasseau, est maintenu rigide par un carton neutre invisible, un cadre vient par-dessus le magazine. Il n'y a pas de passe-partout, ni de fonds au cadre.

8. - Voir le site: http://www.agence-nationale-recherche.fr/suivi-bilan/editions-2013-etanterieures/sciences-et-technologies-de-l-information-et-de-la-communication/contenusnumeriques-et-interactions/fiche-projet-contint/?tx_lwmsuivibilan_pi2\%5BCODE\%5D=ANR-12CORD-0031 [consulté le 24/08/2018].

9. - CNAM : Conservatoire national des arts et métiers.

10. - IGN : Institut géographique national. 


\section{RÉSUMÉS}

Malgré son nom, le musée Nicéphore Niépce (Chalon-sur-Saône) ne se consacre pas exclusivement à l'invention de la photographie. Une histoire singulière de la photographie y est abordée, de Niépce aux dernières avancées du numérique, de la photographie plasticienne à la photographie amateur, de la production industrielle au photoreportage. À ce titre, le musée Nicéphore Niépce a constitué depuis son origine une collection riche de plus de trois millions de phototypes, 6000 appareils photographiques, 30000 livres de photographies et 40000 revues photographiques, revues illustrées par la photographie ou sur la photographie. La photographie de presse est un des axes majeurs d'enrichissement, d'étude, d'exposition et de diffusion développé par le musée Nicéphore Niépce. Si quelques corpus de tirages de presse étaient déjà présents dans les collections (Chusseau-Flaviens, agence Rol), le musée a depuis vingt ans systématiquement collecté des revues photographiques (l'intégralité des numéros à chaque fois) et acquis des archives de photographes indépendants travaillant pour la presse (totalité des négatifs, planches-contacts, tirages de lecture, justificatifs de publication, carnets, etc.). Après inventaire, analyse, documentation et numérisation, le musée Nicéphore Niépce valorise ces collections par des expositions qui ne sont plus des moments de délectation d'œuvres déjà connues mais des expériences d'immersion complexe, de découverte et d'exploration de la démarche d'un auteur. Le musée a également développé des dispositifs multimédias intégrés à son parcours d'exposition ou en ligne, de manière à rendre compte de l'histoire des revues, de leur contexte de production, des spécificités de leur maquette et de leur réception par les lecteurs de l'époque.

In spite of its name, the Nicéphore Niépce museum at Chalon-sur-Saône is not exclusively devoted to the invention of photography. The whole history of photography is dealt with by the museum, from Niépce to the most recent advances in the realm of digital photography, from art photography to amateur photography and from industrial production to photo-reportage. Since its creation in 1972, the museum has built up a rich collection of more than three million photos, 6,000 cameras, 25,000 photography books and 40,000 magazines of, or about, photography. Press photos represent one of the main sectors in which the collections are growing richer and in which the museum is undertaking studies and organising exhibitions. Some collections of press photo prints were already included in the museum's reserves (Chusseau-Flaviens, Rol agency) but over the last twenty years the museum has become more systematic in its prospection for photo magazines (complete collections whenever possible) and the archives of photographers working for the press (complete collections where possible, including negatives, contact sheets, layout prints, publication documents, notebooks, etc.) After their cataloguing, analysis, documentation and digitisation, the museum promotes these collections by organising exhibitions. These are not merely moments of delectation amongst pictures that are already familiar, but complex experiences of immersion, exploration and discovery of a particular photographer's ways of seeing. The museum has also developed multimedia displays which are integrated into the exhibitions or made available online, explaining the history of a magazine, the context of its publication, the specificities of its design and its appreciation by the public of its time. 
INDEX

Keywords : press photography, exhibition, museum, multimedia, magazine

Mots-clés : photographie de presse, exposition, musée, multimédia, magazine

\section{AUTEUR}

\section{SYLVAIN BESSON}

Directeur des collections, Musée Nicéphore Niépce, Chalon-sur-Saône sylvain.besson@chalonsursaone.fr 